\title{
Cor triatriatum dexter associated to Ebstein anomaly with tricuspid double lesion and atrial septal defect
}

\section{Cor triatriatum dexter asociado a anomalía de Ebstein con doble lesión tricúspidea y comunicación interatrial}

\author{
Ricardo Alvarez-Santana ${ }^{1,2}$, Jesus A. Garcia-Diaz ${ }^{1,3}$, Mara Escudero-Salamanca ${ }^{1,4}$, \\ Roberto Cano-Zarate ${ }^{5}$, and Nilda Espinola-Zavaleta ${ }^{1,6 *}$
}

${ }^{1}$ Department of Nuclear cardiology, National Institute of Cardiology Ignacio Chávez, Mexico City; ${ }^{2}$ Institute of Medical Sciences, Autonomous University of Ciudad Juarez, Ciudad Juarez, Chihuahua; ${ }^{3}$ Academic Unit of Medicine, Autonomous University of Nayarit, Tepic-Nayarit; ${ }^{4}$ Mexican Faculty of Medicine, La Salle University, Mexico City; ${ }^{5}$ Department of Magnetic Resonance, National Institute of Cardiology Ignacio Chávez, Mexico City; ${ }^{6}$ Department of Echocardiography, ABC Medical Center, I.A.P, Mexico City, Mexico

Female 23-year-old patient with heart murmur diagnosed in the first year of life. She attended to our institute with progressive dyspnea and palpitations of 7-month evolution. Physical examination showed perioral and distal cyanosis with digital clubbing, oxygen saturation of $79 \%$, jugular plethora, arrhythmic heart sounds of upper limbs, fixed second heart sound, systolic tricuspid murmur, and edema. Electrocardiogram and 24 hour Holter monitoring showed atrial fibrillation and right bundle branch block. Transthoracic echocardiogram revealed Ebstein anomaly with moderate displacement of tricuspid septal leaflet (46\%), atrialized right ventricle of $37 \%$, functional ventricle of $63 \%(A)$, double tricuspid lesion with severe regurgitation, tricuspid area of $2.26 \mathrm{~cm}^{2}(\mathrm{~B}, \mathrm{C})$, ostium secundum-atrial septal defect (diameter - $19 \mathrm{~mm}$ ) with right-to-left shunt (D), infundibular dilation (E), non-obstructive cor triatriatum dexter $(F, G)$, and decreased left ventricular ejection fraction (LVEF - 30\%). Cardiac magnetic resonance showed biventricular systolic dysfunction (LVEF: $26 \%$, right ventricular ejection fraction: $19 \%$ ) and fibrosis of the right atrium, interventricular septum and in the sites of septal and posterior leaflets adherence to the right ventricle, $(\mathrm{H}, \mathrm{I})$ (Fig.1).

The patient received medical treatment and oxygen with improvement on clinical symptoms and oxygen saturation $-94 \%$. She was not considered a candidate for surgical treatment. Actually, she continues her follow-up in the outpatient clinic, and she is in functional NYHA Class II.

Cor triatriatum dexter is an extremely rare congenital heart disease with a prevalence of $0.01 \%^{1}$. In this $a b$ normality, the right atrium is divided into two parts by a membrane, resulting on the lack of regression of the right valve of sinus venosus and it is usually associated with other congenital anomalies ${ }^{2}$.

The multimodality imaging allowed us to establish the precise diagnosis and make therapeutic decisions.
Correspondence:

*Nilda Espinola-Zavaleta

E-mail: niesza2001@ hotmail.com
Available online: $21-12-2020$

Date of reception: 30-07-2020

DOI: 10.24875/ACM.200003671
Arch Cardiol Mex. 2021;91(4):508-509 www.archivoscardiologia.com 1405-9940 / @ 2020 Instituto Nacional de Cardiología Ignacio Chávez. Published by Permanyer. This is an open access article under the CC BY-NC-ND license (http://creativecommons.org/licenses/by-nc-nd/4.0/). 


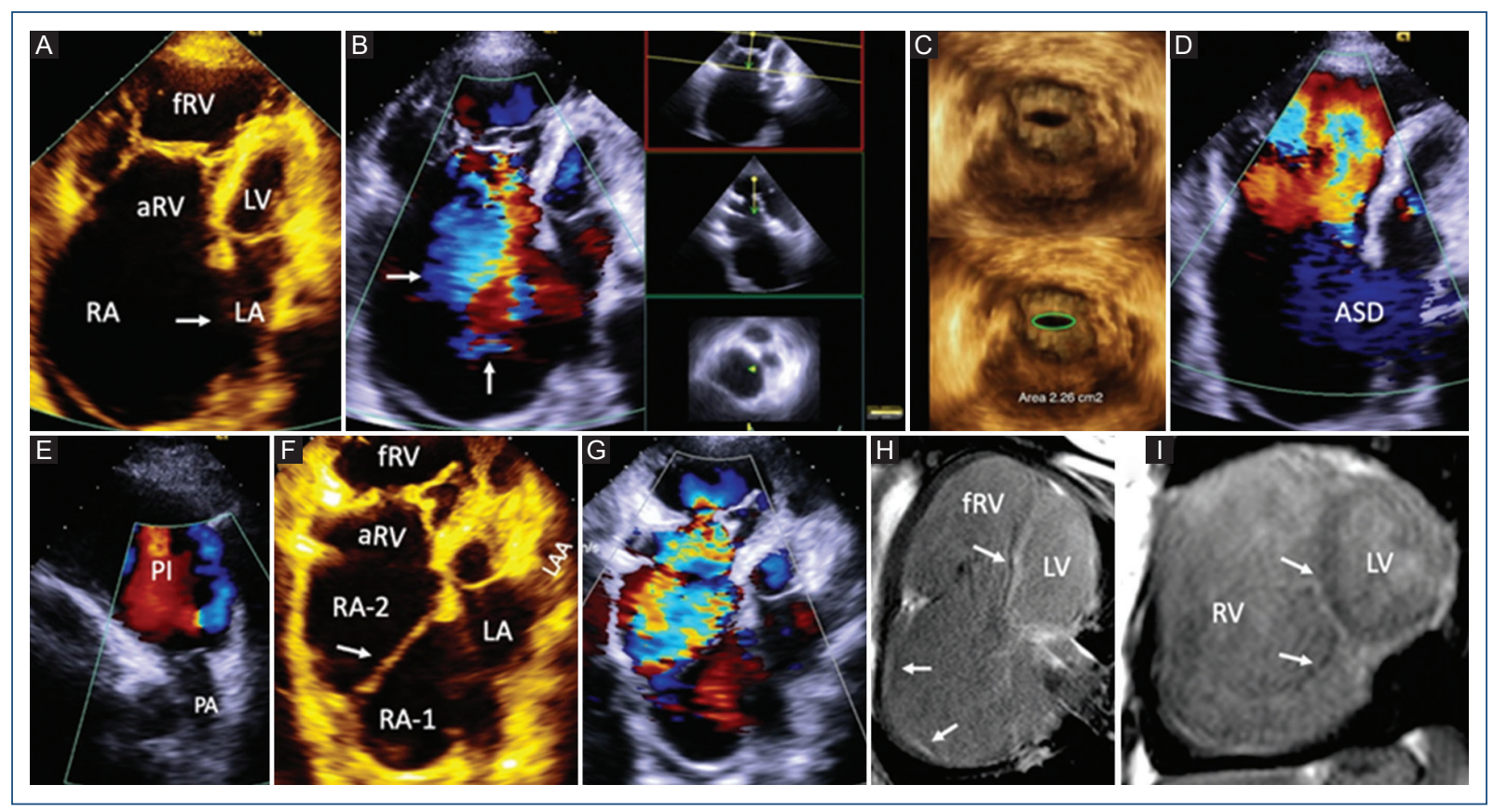

Figure 1. Transthoracic 2-D and 3-D echocardiography and cardiac magnetic resonance. A: Four-chamber view showing moderate Ebstein's anomaly with atrial septal defect (arrow). B: Severe tricuspid regurgitation (arrows). C: 3D echocardiogram showing tricuspid stenosis area of $2.26 \mathrm{~cm}^{2}$. D: Atrial septal defect with right to left shunt. E: Infundibular dilation and normal pulmonary valve. F: Modified four chamber view showing the membrane (arrow) dividing the right atrium in two chambers: RA1 and RA2. G: Color Dopplerrevealed severe tricuspid regurgitation and non-obstructive membrane. H: Four-chamber view and I: short axis of both ventricles with the right atrial and septal fibrosis, also fibrosis in the sites of septal and posterior leaflets adherence to the right ventricle, (arrows). RA: right atrium; aRV: atrialized right ventricle; fRV: functional right ventricle; LA: left atrium; LV: left ventricle; ASD: atrial septal defect; PI: pulmonary infundibulum; PA: pulmonary artery; RA-1: right atrium 1; RA-2: right atrium 2; LAA: left atrial appendage.

\section{Funding}

No financial support.

\section{Conflicts of interest}

None.

\section{Ethical disclosures}

Protection of human and animal subjects. The authors declare that no experiments were performed on humans or animals for this study.
Confidentiality of data. The authors declare that they have followed the protocols of their work center on the publication of patient data.

Right to privacy and informed consent. The authors declare that no patient data appear in this article.

\section{References}

1. Krasemann Z, Scheld HH, Tjan TD, Krasemann T. Cor triatriatum: short review of the literature upon ten new cases. Herz. 2007;32:506-10.

2. Eroglu S, Yildirir A, Simsek V, Bilgi M, Ozin B, Muderrisoglu H. Cor triatriatum dexter, atrial septal defect, and Ebstein's anomaly in an adult given a diagnosis by transthoracic and transesophageal echocardiography: a case report. J Am Soc Echocardiogr. 2004;17:780-2. 